\title{
Easy-dispersible poly(glycidyl phenyl ether)-functionalized graphene sheets obtained by reaction of "living" anionic polymer chains
}

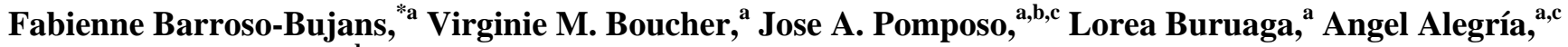 \\ ${ }_{5}$ and Juan Colmenero ${ }^{\mathrm{a}, \mathrm{c}, \mathrm{d}}$
}

Excellent dispersion of functionalized graphene (FG) sheets in polystyrene was achieved relying in the reaction of "living" poly(glycidyl phenyl ether) chains onto graphene sheets. The ${ }_{10}$ physical aging of polystyrene was substantially accelerated by the presence of FG sheets at low filler content, retaining film transparency and increasing the electrical conductivity.

Free standing polymer/graphene composite thin films are envisioned as hybrid nanomaterials in which the best of both 15 components could be combined to obtain added value (e.g., barrier, thermal, electrical) properties while retaining a high level of transparency. ${ }^{1}$ Maintaining film transparency and providing appropriate electrical conductivity $(\sigma)$ is crucial for industrial applications such as: i) antistatic bags that require an electrical 20 conductivity level in the range $\sigma \sim 10^{-12}-10^{-6} \mathrm{~S} / \mathrm{cm}$, and ii) electrostatic charge dissipation (ESD) cans to prevent damage of microelectronics components, requiring $\sigma \sim 10^{-6}-10^{-4} \mathrm{~S} / \mathrm{cm}^{2}$

Exfoliation and reduction of graphite oxide by thermal treatment provide graphene sheets with significant electrical conductivity ${ }_{25}\left(\sim 10^{1} \mathrm{~S} / \mathrm{cm}\right)$ and hold promise in various applications such as biosensing or bioelectronics. Moreover, the thermally expanded / reduced graphite oxide (here denoted as G) sheets contain residual epoxide, carbonyl and hydroxyl groups which greatly facilitate their functionalization (i.e., grafting of polymer chains) 30 and dispersion in polymeric matrices (e.g., polystyrene, PS). ${ }^{1}$ Recent reports have demonstrated both noncovalent (e.g., $\pi-\pi$ stacking, ionic interactions) and covalent (e.g., diazonium, organosilane-based) functionalization of $\mathrm{G}$ sheets. ${ }^{3}$ However, grafting of polymer chains selectively at existing defects to avoid 35 adversely affecting electrical transport is extremely rare.

In this communication, we report an efficient route to obtain $G$ sheets functionalized at residual electrophilic sites with oligomeric poly(glycidyl phenyl ether) (PGPE) chains. The obtained PGPE-functionalized graphene sheets, here denoted as ${ }_{40} \mathrm{FG}$, are shown to disperse efficiently in PS by means of electrical conductivity, optical transparency and physical aging experiments. Thin films of PS/FG nanocomposites were found to retain transparency at low filler content $(<0.5 \mathrm{wt} \%)$ and to exhibit significant electrical conductivity and accelerated physical 45 aging as compared to neat PS.

Hence, inspired by both end-group functionalization of living anionic polymers ${ }^{4}$ and anionic deactivation of carbonyl groups ${ }^{5}$ we hypothesized that living anionic chains could react with residual electrophilic groups of $\mathrm{G}$ sheets (e.g. ketone, epoxy) 50 when added to a polymerization medium containing such chains (Fig. 1(I)). In this sense, we selected the ring opening polymerization (ROP) of glycidyl phenyl ether (GPE) in bulk at $60{ }^{\circ} \mathrm{C}$ initiated by tetrabutylammonium fluoride $(\mathrm{TBAF})^{6}$ as an easy and robust technique to obtain monodisperse oligomeric 55 PGPE chains with living alkoxy groups at one of the chain ends. Metal-free ROP of GPE is really a facile technique because the initiator is commercially available, the monomer is a common epoxide and the stability of the propagating species during ROP enables a living-like polymerization. ${ }^{6}$ After $6 \mathrm{~h}$ of GPE ROP time 60 (corresponding to living PGPE chains of molar mass 1,900 $\mathrm{g} / \mathrm{mol}$ ) $\mathrm{G}$ sheets were added to the reaction medium and allowed to react for $20 \mathrm{~min}$. The resulting PGPE-functionalized graphene (FG) sheets were purified from free PGPE chains and finally isolated by drying under dynamic vacuum. The amount of PGPE ${ }_{65}$ chains anchored to the graphene sheets was found to be $14 \mathrm{wt} \%$, corresponding, on average, to one grafted PGPE chain every 1000 carbon atoms (see ESI).

Polystyrene nanocomposites filled with 0.1 and $0.5 \mathrm{wt} \%$ FG sheets were prepared by casting from toluene solutions and 70 further solvent drying. PS/FG nanocomposites were compared to nanocomposites prepared under identical conditions by using neat, non-functionalized $\mathrm{G}$ sheets as a control. As illustrated in the transmission electron microscopy (TEM) images of Fig. 1(II), excellent dispersion of FG sheets in the PS matrix was observed 75 as compared to that of nonfunctionalized $G$ sheets, the latter showing the presence of micrometer-sized aggregates as a consequence of the low compatibility of neat $\mathrm{G}$ sheets and PS. This suggests that PGPE-grafting is a prerequisite for easy dispersion of the graphene filler in the polymeric matrix.

${ }_{80}$ Fig. 2(a) illustrates the optical transparency of the PS/FG and $\mathrm{PS} / \mathrm{G}$ nanocomposite thin films as a function of film thickness, as determined by ultraviolet-visible (UV-Vis) spectroscopy at a wavelength of $500 \mathrm{~nm}$ following conventional procedures. ${ }^{7}$ In general, no significant change in transparency was observed in 85 the $300-1100 \mathrm{~nm}$ wavelength range (see ESI). As expected, a certain reduction in optical transparency was observed for PS/FG and PS/G nanocomposites upon increasing both filler content and film thickness. Due to the better dispersion of the FG sheets, a larger transparency loss was found at $0.5 \mathrm{wt} \%$ filler loading for 


\section{Author's Version}

ARTICLE

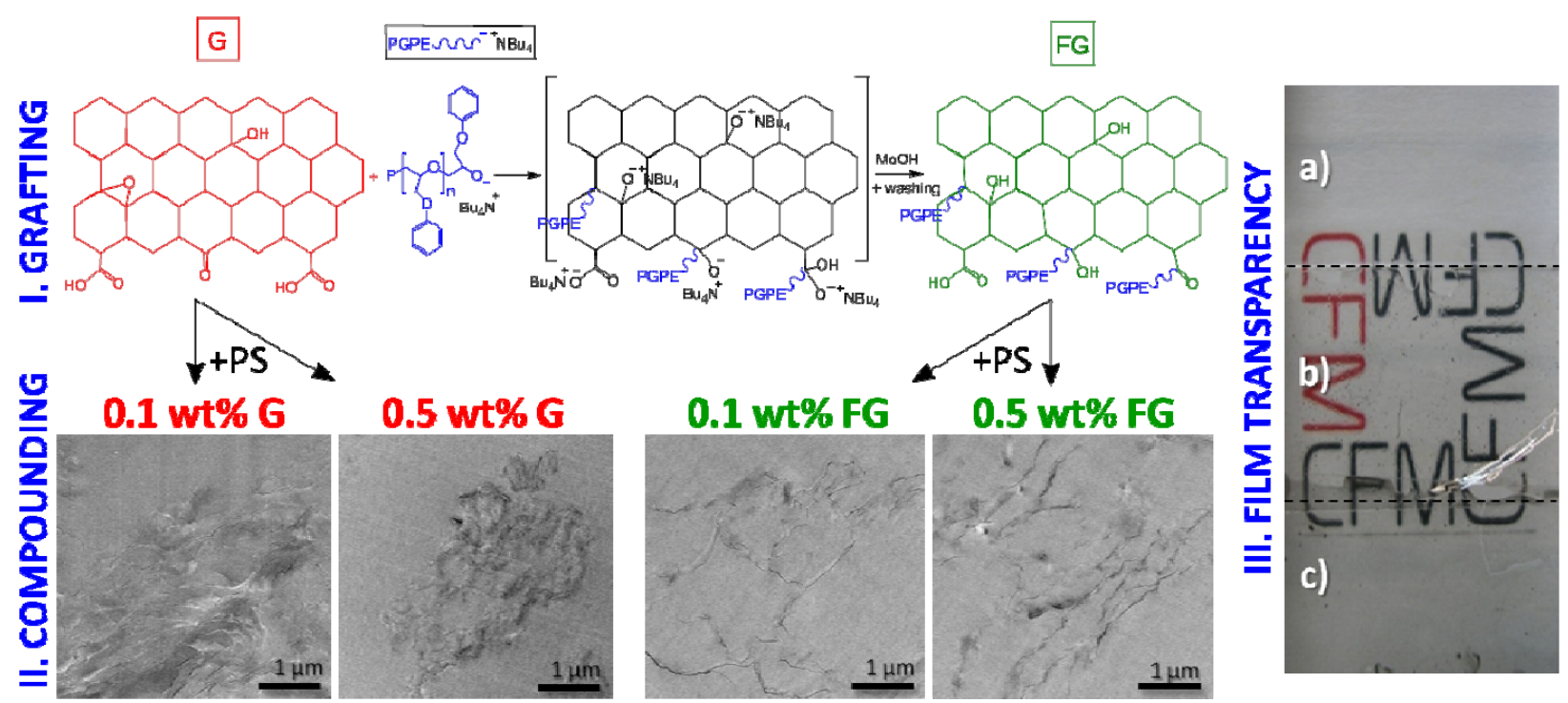

Fig. 1 (I) Scheme of the functionalization of G with PGPE by the simple reaction of alkoxy-terminated "living" PGPE chains with residual electrophilic groups of G. (II) Efficient dispersion of FG sheets (right) in PS as compared to neat G (left) as determined by TEM. The filler content is indicated in each micrograph. (III) Optical images of glass-supported $2.4 \mu \mathrm{m}$ films for (a) neat PS, (b) PS/G and (c) PS/FG nanocomposites with $0.5 \mathrm{wt} \%$ filler content.

5 the former in comparison with the latter, displaying "open" regions where photons are easily transmitted as a consequence of the significant aggregation of the $\mathrm{G}$ sheets (Fig. 1(II-III)). ${ }^{8}$ The optical images of glass-supported $2.4 \mu \mathrm{m}$ films obtained by spin coating (Fig. 1(III)) show the presence of macrosized aggregates 10 in $\mathrm{PS} / \mathrm{G}$ composites, which disappear in the PS/FG material to give rise to a much more homogeneous and darker film. At 0.1 $w t \%$ filler loading, such a different behaviour is hard to detect by $\mathrm{UV}-\mathrm{V}$ is measurements.

Fig. 2(b) shows the electrical conductivity $(\sigma)$ as a function of 15 frequency $(f)$ for PS/FG and PS/G nanocomposites at $343 \mathrm{~K}$ and $423 \mathrm{~K}$ as determined by broadband dielectric spectroscopy (BDS) measurements. In neat PS and PS/G composites, $\sigma$ follows a linear dependence with frequency displaying a slope close to 1 , which is characteristic of insulating materials. However, PS/FG 20 composites display the characteristic behaviour of semiconducting materials with a low frequency plateau and a frequency-dependent conductivity at higher frequencies. ${ }^{9}$ For PS/FG nanocomposites, the frequency-independent direct current conductivity $\left(\sigma_{\mathrm{dc}}\right)$ values can be extracted directly from the 25 plateau at low frequencies as summarized in Table S1 (ESI). The $\sigma_{\mathrm{dc}}$ value of both PS and PS/G composites, for which the plateau was not reached even at the lowest frequencies investigated, is reported in Table S1 (ESI) as $\sigma_{\mathrm{dc}}<10^{-14} \mathrm{~S} / \mathrm{cm}$. The most obvious feature is that PS/FG nanocomposites exhibit significantly higher 30 conductivity values than those of PS and PS/G nanocomposites. The good dispersion of FG sheets in the PS matrix favours the formation of a conducting percolative network at very low critical concentrations, which is highly desirable in order to retain the PS for all nanocomposite samples, especially when increasing the filler content. These results are in agreement with previous works optical transparency of the PS matrix. In particular, the PS/FG 35 nanocomposite at $0.5 \mathrm{wt} \%$ filler loading displays a conductivity value appropriate for ESD applications ${ }^{2}$ with only a minor dependency of $\sigma_{\mathrm{dc}}$ on temperature (Fig. 2(b)). On the contrary, a significant change of $\sigma_{\mathrm{dc}}$ with temperature is observed in Fig. 2(b) for the $\mathrm{PS} / \mathrm{FG}$ nanocomposite with $0.1 \mathrm{wt} \%$ filler loading. In 40 general, differences in the temperature dependency of $\sigma_{\mathrm{dc}}$ are attributed to the different structure of the conductive filler, its loading, and its dispersion state. ${ }^{10}$

Additionally, we have investigated the physical aging of PS/FG and PS/G nanocomposites. Physical aging is known as the slow 45 evolution of thermodynamic properties (e.g., enthalpy, volume) towards equilibrium below the glass transition temperature $\left(\mathrm{T}_{\mathrm{g}}\right)$ of polymer materials. ${ }^{11}$ As this temperature range can coincide with the range of applicability of these materials, physical aging can drastically affect the properties of these systems. Thereby, 50 control of physical aging is needed for obtaining stable materials. The segmental dynamics of PS as determined from BDS was shown to be unaltered by the presence of the FG and G fillers, allowing to perform a comparative study of physical aging at the same aging temperature $\left(\mathrm{T}_{\mathrm{a}}\right)$ for all samples. ${ }^{12}$

${ }_{55}$ This study was carried out by differential scanning calorimetry (DSC) by evaluating the recovered enthalpy after aging at a fixed $\mathrm{T}_{\mathrm{a}}$, during various aging times $\left(\mathrm{t}_{\mathrm{a}}\right)$. At long aging times, a plateau value of the recovered enthalpy $\Delta H(\infty)$ was reached for all samples. Fig. 2(c) displays the distance of the recovered enthalpy ${ }_{60}\left(\Delta \mathrm{H}\left(\mathrm{t}_{\mathrm{a}}\right)\right)$ from the plateau value $(\Delta \mathrm{H}(\infty))$ as a function of aging time after annealing at $343 \mathrm{~K}$. This plot highlights the shorter aging times required to reach the value of $\Delta H(\infty)$ relative to pure ${ }_{65}$ on other polymer nanocomposite systems. ${ }^{12}$ Moreover, at constant filler loading and $\mathrm{T}_{\mathrm{a}}$, a faster achievement of the plateau 


\section{Author's Version}

ARTICLE
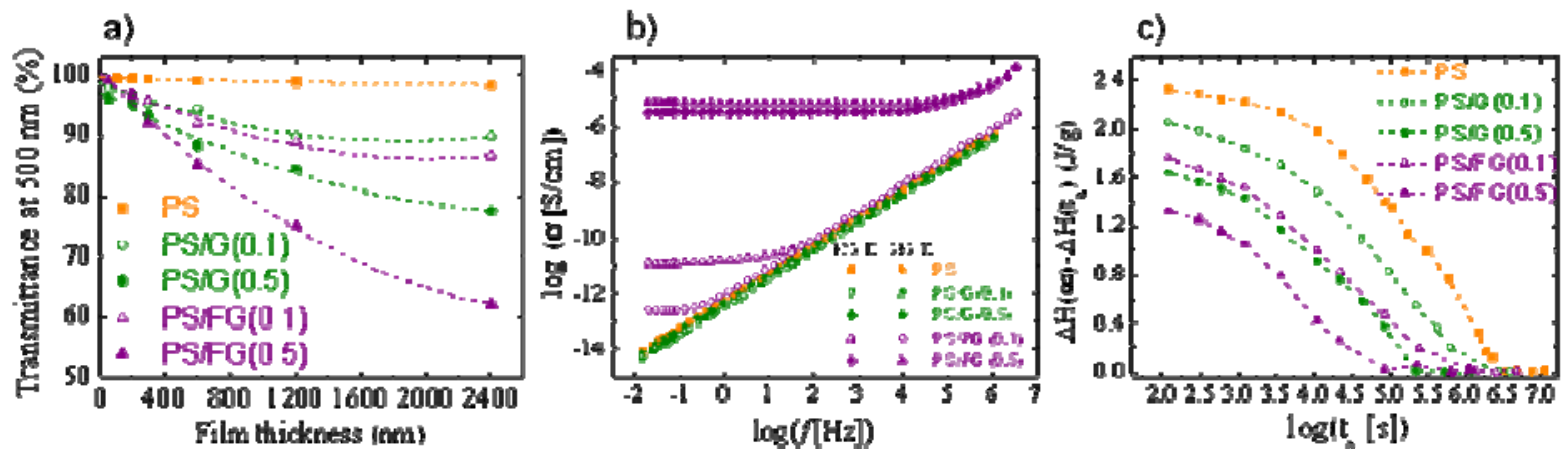

Fig. 2 (a) Transmittance as a function of film thickness for neat PS, PS/G and PS/FG nanocomposites at a wavelength of 500 nm, as determined by UVVis spectroscopy. (b) Electrical conductivity $(\sigma)$ as a function of frequency ( $\mathrm{f}$ ) as determined by BDS. (c) Distance of the recovered enthalpy $(\Delta \mathrm{H}$ (ta)) to the limiting "equilibrium" value $(\Delta \mathrm{H}(\infty))$ as a function of aging time at $343 \mathrm{~K}$, as determined by DSC. The filler content (in wt\%) is indicated in brackets.

${ }_{5}$ is observed for PS/FG composites in comparison with $\mathrm{PS} / \mathrm{G}$ composites. Complementary physical aging experiments carried out on ternary PS/PGPE/G blends showed that their enthalpy recovery was roughly similar to that of $\mathrm{PS} / \mathrm{G}$ nanocomposites at equivalent filler loading (see ESI). According to previous 10 findings showing the key role of the (filler area) / (polymer volume) ratio in the acceleration of the aging process in polymer nanocomposites, ${ }^{13}$ this observation also points to an excellent dispersion of the graphene sheets in the PS/FG nanocomposites. Astonishingly, the plateau value of the enthalpy recovery is ${ }_{5}$ reached in about only 1 day for PS/FG nanocomposites $(0.5 \mathrm{wt} \%$ filler loading) versus more than 1 month for the neat PS matrix by annealing at $343 \mathrm{~K}$, which decreases to $3.5 \mathrm{~h}$ versus 5 days at 358 $\mathrm{K}$ and which even reduces to $40 \mathrm{~min}$ versus 14 hours in neat PS by annealing at $366 \mathrm{~K}$ (see ESI).

20 In conclusion, a simple and highly efficient method for decoration of thermally expanded/reduced graphite oxide sheets with poly(glycidyl phenyl ether) chains inspired from terminalcapping of living anionic polymers has been demonstrated. This methodology could pave the way to $G$ sheet decoration onto ${ }_{25}$ defect sites with other "living" anionic polymers. The resulting FG sheets displayed an excellent degree of dispersion in PS and an accelerated physical aging in comparison with neat, nonfunctionalized $\mathrm{G}$ sheets as demonstrated by a combination of techniques including TEM, UV-Vis spectroscopy, BDS and DSC. 30 An interesting implication of the faster achievement of limiting values of thermodynamic properties in PS/FG nanocomposites is the potential reduction of technological problems arising from changes in material properties during lifetime.

The authors acknowledge the support of the Spanish Ministry of ${ }_{35}$ Education (CSD2006-00053), the Basque Government (IT-43607), and SGIker UPV/EHU for TEM and NMR facilities. FBB and VB acknowledge the JAE-Doc contract from CSIC.

\section{Notes and references}

${ }^{a}$ Centro de Física de Materiales-Material Physics Center (CSIC40 UPV/EHU),Paseo Manuel Lardizábal 5, 20018 San Sebastián, Spain.
Fax: +34 943015800; Tel: +34 94301 8793; E-mail: fbarroso@ehu.es (F Barroso-Bujans)

${ }^{b}$ IKERBASQUE-Basque Foundation for Science, Alameda Urquijo 36,48011, Bilbao, Spain

$45^{c}$ Departamento de Física de Materiales, Universidad del País Vasco (UPV/EHU) Apartado 1072, 20080 San Sebastián, Spain

${ }^{d}$ Donostia International Physics Center, Paseo Manuel Lardizábal 4, 20018 San Sebastián, Spain

$\dagger$ Electronic Supplementary Information (ESI) available: [Materials, 50 characterization techniques and methods.]. See DOI: 10.1039/b000000x/

1 (a) R. Verdejo, M. M. Bernal, L. J. Romasanta and M. A. LopezManchado, J. Mater. Chem., 2011, 21, 3301; (b) H. Kim, A. A. Abdala and C. W. Macosko, Macromolecules, 2010, 43, 6515; (c) Y. Zhu, S. Murali, W. Cai, X. Li, J. W. Suk, J. R. Potts and R. S. Ruoff, 55 Adv. Mat., 2010, 22, 3906. (d) J. R. Potts, D. R. Dreyer, C.W. Bielawski and R. S. Ruoff, Polymer, 2011, 52, 5.

2 B. W. Steinert and D. R. Dean, Polymer, 2009, 50, 898.

3 (a) M. Steenackers, A. M. Gigler, N. Zhang, F. Deubel, M. Seifert, L. H. Hess, C. H. Y. X. Lim, K. P. Loh, J. A. Garrido, R. Jordan, M. $60 \quad$ Stutzmann and I. D. Sharp, J. Am. Chem. Soc., 2011, 133, 10490; (b) H. J. Salavagione, G. Martínez and G. Ellis, Macromol. Rapid Commun., 2011, doi:10.1002/marc.201100527.

4 M. Arnould, M. Polce, R. Quirk and C. Wesdemiotis, Int. J. Mass Spectrom., 2004, 238, 245.

655 (a) M. Klapper, T. Wehrmeister and K. Müllen, Macromolecules, 1996, 29, 5805; (b) J. Li and M. Gauthier, Macromolecules, 2001, 34, 8918.

6 H. Morinaga, B. Ochiai and T. Endo, Macromolecules, 2007, 40, 6014.

7 J. G. Smith-Jr, D. M. Delozier, J. W. Connell and K. A. Watson, Polymer, 2004, 45, 6133.

708 T. Kashiwagi, J. Fagan, J. F. Douglas, K. Yamamoto, A. N. Heckert, S. D. Leigh, J. Obrzut, F. Du, S. Lin-Gibson, M. Mu, K. I. Winey and R. Haggenmueller, Polymer, 2007, 48, 4855.

9 A. Linares, J. C. Canalda, M. E. Cagiao, M. C. Garcia-Gutierrez, A. Nogales, I. Martin-Gullon, J. Vera and T. A. Ezquerra, Macromolecules, 2008, 41, 7090.

10 M. T. Connor, S. Roy, T. A. Ezquerra and F. J. Baltá Calleja, Phys. Rev. B, 1998, 57, 2286.

11 I. M. Hodge, J. Non-Cryst. Solids, 1994, 169, 211.

12 (a) V. M. Boucher, D. Cangialosi, A. Alegria, J. Colmenero, I. $80 \quad$ Pastoriza-Santos and L. M. Liz-Marzan, Soft Matter, 2011, 7, 3607; (b) V. M. Boucher, D. Cangialosi, A. Alegria and J. Colmenero, Macromolecules, 2010, 43, 7594.

13 D. Cangialosi, V. M. Boucher, A. Alegria and J. Colmenero, J. Chem. Phys., 2011, 135, 014901. 